\title{
Associação Entre Doença Periodontal Materna e Parto Pré-termo e Baixo Peso ao Nascimento
}

\author{
OLIVEIRA, Alcione Maria Soares Dutra de * $_{*}^{*}$ \\ OLIVEIRA, Peterson Antônio Dutra de ***** $_{* *}^{*}$ \\ COSTA, Fernando de Oliveira $a_{* * *}$ \\ MANZI, Flávio Ricardo ${ }_{* * * * *}$ \\ COSSO, Maurício Greco
}

\begin{abstract}
RESUMO
Este estudo teve como objetivo avaliar a associação da condição periodontal materna na ocorrência de parto pré-termo e baixo peso ao nascimento de (PTBPN). Participaram do estudo 236 mulheres, com a média de 25 anos de idade e características socioeconômicas semelhantes, que deram à luz em uma instituição de saúde pública de Belo Horizonte (MG - Brasil). Os dados maternos, obstétricos e pediátricos foram obtidos nos prontuários médicos. $O$ exame periodontal foi realizado no período de internação pós-parto, em seis sítios ao redor de todos os dentes presentes e incluiu a avaliação dos parâmetros clínicos profundidade de sondagem (PS), nível clínico de inserção (NIC) e sangramento à sondagem (SS). Para a análise considerou-se como caso de doença periodontal a presença de um sítio com PS e NIC e" $4 \mathrm{~mm}$. Não houve diferenças significantes quando da análise das variáveis raça, escolaridade, prematuridade prévia, diabetes, infecção geniturinária, consumo de álcool, hábito de fumar e doença periodontal. $O$ teste Qui-quadrado mostrou significância estatística para os fatores de risco hipertensão ( $p=0,016)$ e consumo de drogas ilícitas $(p=0,017)$. Já o teste $\mathrm{t}$ Student mostrou significância estatística para a variável idade $(p=0,010)$. Na análise multivariada somente o consumo de drogas ilícitas mostrou-se significativo: elevou de $25 \%$ para $66,7 \%$ a ocorrência de PTBPN. Esses resultados demonstraram que a condição periodontal materna não apresentou associação estatisticamente significativa com a ocorrência de PTBPN $(p=0,364)$.
\end{abstract}

\section{PALAVRAS-CHAVE:}

Doença periodontal, efeitos adversos. Parto pré-termo. Baixo peso ao nascimento. Gravidez, fator de risco.

\section{INTRODUÇÃO}

Evidências científicas advindas de estudos clínicos epidemiológicos realizados a partir dos anos 1980 apontam para uma provável associação da infecção periodontal na ocorrência do parto pré-termo e baixo peso ao nascimento (Mc-CORMICK, 1985; GIBBS et al. 1992; DASANAYAKE, 1998; OFFENBACHER et al. 1996; OFFENBACHER et al., 1998; SCANNAPIECO et al., 2003; RADNAI et al., 2004; KHADER e TA' ANI, 2005).

Collins et al. (1994; 1995) demonstraram que a aplicação de injeções com periodontopatógenos, como $P$. gingivalis, nas fêmeas prenhas causou uma redução significativa no peso dos filhotes em as- sociação com o aumento nos níveis de fator de necrose tumoral (TNF) e PGE, no útero dos animais.

Offenbacher et al. (1998) realizaram análise microbiológica e encontraram níveis significativamente aumentados de periodontopatógenos nas mães com PTBPN em relação ao grupo controle. Hill (1998) relaciona espécies bacterianas, características da flora subgengival, como participantes de quadros de infecção vaginal e ocorrência de pré-termo, com isolamento de Fusobacterium nucleatum e Capnocytophaga do líquido amniótico de mulheres com PTBPN. Mitchell-Lewis et al. (2001) identificaram, na placa subgengival de mães que tiveram parto pré-termo, níveis significativamente maiores de Bacteróides forsythus e Campylobacter rectus além de contagens elevadas para outras espécies examinadas.

Níveis de PGE significativamente maiores no fluido geengival de mulheres com parto prematuro foram observados por Offenbacher et al. (1998). Hasegawa et al. (2003) demonstraram que as mulheres com ameaça de parto pré-termo apresentaram uma pior condição periodontal e níveis de IL-8 e IL-1ß séricos elevados em comparação com aquelas com parto a termo. Dasanayake et al. (2001) detectaram maiores níveis sorológicos de IgG para Porphyromonas gingivalis, no

* Professora Adjunta de Periodontia e Implantodontia da FOPUC Minas. Especialista, Mestre e Doutoranda em Periodontia pela FOUFMG.

** Professor de Periodontia e Implantodontia da FOPUC Minas. Especialista e Mestre em Periodontia pela FOUFMG. Doutorando em Implantodontia pela FO São Leopoldo Mandic. *** Mestre em Periodontia pela FOUFMG. Doutor em Ciência Animal pela Escola de Veterinária da Universidade Federal de Minas Gerais. Professor da FOPUC Minas e FOUFMG **** Professor Adjunto em Radiologia e DTM da FOPUC Minas. Professor colaborador em Radiologia da FO de Piracicaba - UNICAMP.

Mestre e Doutor em Radiologia pela FO de Piracicaba - UNICAMP ***** Professor de Cirurgia e Implantodontia da FOPUC Minas. Mestre e Doutorando em Implantodontia pela FO São Leopoldo Mandic. 
segundo trimestre da gravidez, nas mães que deram à luz crianças de baixo peso.

Vários estudos analisaram a condição clínica periodontal e observaram que as mulheres que deram à luz bebês prematuros e de baixo peso apresentaram maior gravidade de doença periodontal (DASANAYAKE, 1998; DAVENPORT et al., 1998; JEFFCOAT et al., 2001; OFFENBACHER et al., 1998; LOPES et al., 2002a JARJOURA et al., 2005) Já os estudos de Mitchell-Lewis et al. (2001), Davenport et al. (2002), Moore et al. (2005) e Noack et al. (2005) não demonstraram diferença significativa entre a condição clínica periodontal e a ocorrência de parto pré-termo.

Com relação ao impacto do tratamento periodontal no curso da gestação, os resultados apontam para um efeito benéfico da intervenção periodontal, ou seja, redução do risco de ocorrência do parto pré-termo (JEFFCOAT et al., 2003; MITCHELL-LEWIS et al., 2001; LOPES et al., 2002b; LOPES et al., 2005).

O presente estudo do tipo transversal teve o objetivo de avaliar a associação entre a doença periodontal materna com o parto pré-termo e baixo peso ao nascimento.

\section{MATERIAL E MÉTODO}

A população do estudo constou de 236 mulheres, com idade entre 18 e 36 anos, que deram à luz em uma mesma instituição pública de saúde; a MaternidadeEscola Hilda Brandão da Santa Casa de Misericórdia de Belo Horizonte (MG, Brasil). Quanto à cor da pele 27,2\% eram melanodérmicas, $48,5 \%$ feodérmicas e $24,3 \%$ leucodérmicas.

O projeto de pesquisa foi aprovado pelo Comitê de Ética em Pesquisa tanto da Pontifícia Universidade Católica de Minas Gerais quanto da Santa Casa de Misericórdia de Belo Horizonte.

Foram incluídas no estudo mulheres com gestação única, que deram à luz recém-nascidos vivos, resultantes de gestação a termo ou parto pré-termo espontâneo. Os fatores de exclusão foram: (a) gestação múltipla; (b) risco para a ocorrência de endocardite infecciosa; (c) uso de antibióticos nos últimos três meses gestação; (d) cardiopatias; (e) anormalidades placentárias; e (f) interrupção da gestação por motivos maternos e ou fetais diversos. Das 236 mulheres convidadas, quatro se recusaram a participar do estudo, e uma apresentou fator de risco para endocardite infecciosa.

Obtiveram-se dados referentes a aspectos demográficos, história médica, condição periodontal materna, além de dados pediátricos. A partir de entrevista individual e do prontuário médico, coletaram-se informações sobre os fatores de risco clássicos para a ocorrência de parto pré-termo e nascimento de crianças de baixo peso, tais como raça, idade, história pregressa de $\mathrm{PTBPN}$, infecção geniturinária, hipertensão, hábito de fumar, consumo de bebidas alcoólicas e drogas ilícitas durante a gestação.

\section{Avaliação da condição clínica periodontal}

As pacientes foram submetidas a um exame periodontal que incluiu avaliação dos parâmetros clínicos de sangramento à sondagem ( $\mathrm{SS}$ ), profundidade de sondagem (PS) e nível de inserção clínica (NIC). Para o exame periodontal utilizou-se uma sonda manual UNC-15 probe, Hu-FriedyÒ. Os critérios clínicos foram mensurados através de sondagem circunferencial, em seis sítios (mésio, mediano e disto), ao redor de todos os dentes presentes nas superfícies vestibular e lingual/palatina.

Todos os exames periodontais foram realizados pelo mesmo examinador devidamente calibrado para os critérios do estudo. Os valores de concordância obtidos a partir do teste KAPPA foram $\mathrm{K}=0,7614$ para o critério $\mathrm{PS}$ e $\mathrm{K}=\mathbf{0 , 8 3 0 2}$ para o critério NIC.

No momento do exame clínico periodontal, foram considerados os seguintes critérios de exclusão para os elementos dentais: (a) presença de, pelo menos, 18 dentes naturais; (b) remanescentes radiculares; (c) dentes com restaurações insatisfatórias que impossibilitassem o exame periodontal de forma adequada; (d) dentes com processos agudos; (e) os terceiros molares. Com base nos parâmetros clínicos de SS, PS e do NIC, determinaram-se o diagnóstico e a gravidade da doença periodontal.

Na caracterização da periodontite, a presença de apenas um sítio com profundidade de sondagem (PS) ${ }^{3} 4 \mathrm{~mm}$ e com perda de inserção clínica (PIC) ${ }^{3} 4 \mathrm{~mm}$ foi suficiente para a definição de um casodoença periodontal invasiva. Na ausência de evidência clínica de perda de inserção e profundidade de sondagem significativa, a presença de, no mínimo, quatro sítios com sangramento à sondagem caracterizou um caso de gengivite.

A fim de classificar a gravidade da periodontite, adotaram-se os parâmetros da Academia Americana de Periodontia (American Academy of Periodontology, 2000):

a. periodontite de leve a moderada: profundidade de sondagem (PS) até $6 \mathrm{~mm}$ / perda de inserção clínica (PIC) até 4 mm;

b. periodontite avançada: profundidade de sondagem (PS) $>6 \mathrm{~mm} /$ perda de inserção clínica (PIC) $>4 \mathrm{~mm}$.

A categorização da extensão da gengivite e da periodontite obedeceu aos seguintes critérios:

a. doença localizada: até $30 \%$ áreas envolvidas;

b. doença generalizada: $>30 \%$ áreas envolvidas.

\section{Definição do desfecho gestacional}

A idade gestacional foi determinada a partir da avaliação do pediatra por meio do teste de Capurro.

A população do estudo foi dividida em dois grupos de acordo com a definição da Organização Mundial de Saúde (WORLD HEALTH ORGANIZATION, 1987):

a. Grupo I: pacientes que deram à luz em idade gestacional normal ( 37 semanas completas e menos que 42 semanas);

b. Grupo II: pacientes que deram à luz em parto pré-termo ( $<37$ semanas) e/ ou crianças com baixo peso ao nascimento $(<2500 \mathrm{~g})$.

\section{Análise estatística}

$\mathrm{Na}$ análise univariada utilizaram-se os testes Qui-quadrado, Exato de Fisher e $\mathrm{t}$ de Student como forma de avaliar os fatores de interesse na ocorrência de prétermo e/ou baixo peso corporal ao nascimento. Além disso, a fim de ajustar os diversos fatores de risco para ocorrência do PTBPN, aplicou-se a análise de regressão logística multivariada e consideraram-se todos os resultados significativos para uma probabilidade de significância inferior a $5 \%(p<0,05)$. O software utilizado foi o Statistical Package for Social Sciences (versão 9.0).

\section{RESULTADOS}

A prevalência de PTBPN na amostra foi de $26,7 \%$; a de partos a termo foi de $73,3 \%$. Diagnosticou-se a condição saúde periodontal em $22,9 \%$ das mulheres: a gengivite ocorreu em $41 \%$, isto é, $24,5 \%$ na forma localizada e $16,5 \%$ na forma generalizada. Identificou-se a periodontite em $36,1 \%$ da amostra: $34,8 \%$ na fase de inicial a moderada e $1,3 \%$ na forma avançada da doença. Nas mulheres com saúde periodontal, houve $20,4 \%$ de casos de PTBPN; 30,9\% entre as mulheres com diagnóstico de gengivite, e 25,9\% de casos no grupo com periodontite. Vale ressaltar que não se constatou diferença significativa $(p=0,364)$ entre os grupos com saúde e os grupos com doença peri- 


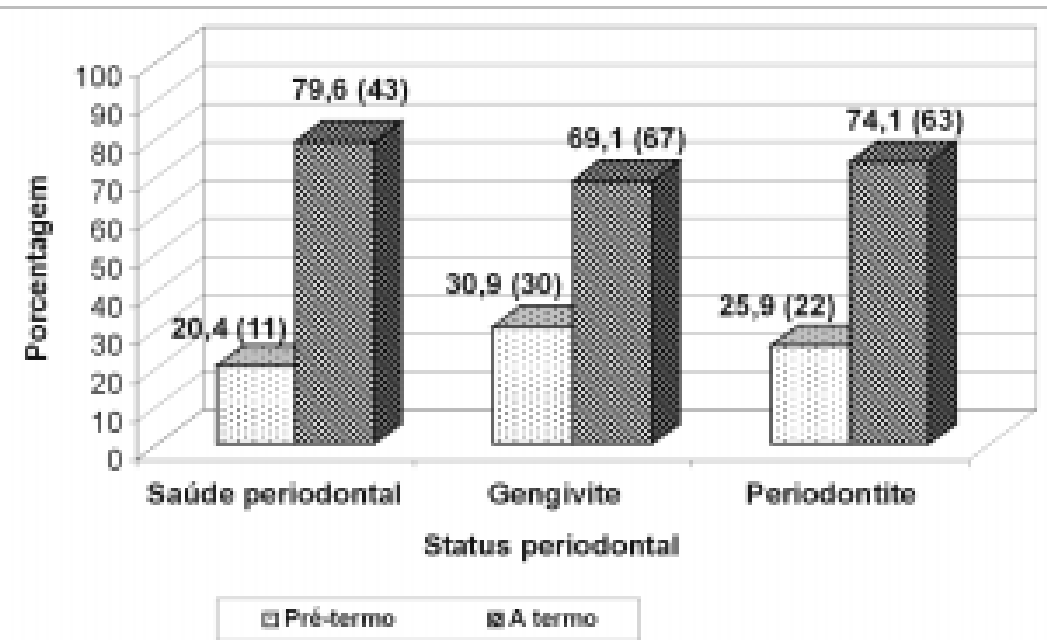

GRÁFICO 1 - Caracterização da amostra quanto à ocorrência de PTBPN considerando-se o status periodontal

Nota: A probabilidade de significância refere-se ao teste Qui-quadrado.

odontal quanto à ocorrência de PTBPN (GRAF. 1).

A condição periodontal em relação à ocorrência de PTBPN também foi analisada dividindo a amostra em dois grupos: o primeiro era constituído de mulheres com saúde periodontal; o segundo, de gestantes que apresentavam sinais de processo inflamatório periodontal (gengivite / periodontite) independentemente da gravidade da doença. Os resultados demonstraram que nas mulheres com diagnóstico de saúde periodontal ocorreram 20,4\% casos de PTBPN e nas pacientes com gengivite e/ou periodontite $28,6 \%(p=0,232)$.

Avaliação de fatores de risco clássicos para ocorrência de PTBPN

A análise estatística dos fatores de risco clássicos para a ocorrência de PTBPN realizada através do Teste Qui-quadrado não demonstrou diferenças significativas para os fatores tabagismo, história pregressa de PTBPN, infecção geniturinária e consumo de bebidas alcoólicas. Porém, as variáveis hipertensão e consumo de drogas ilícitas durante a gravidez apresentaram valores significativos. Verificou-se também que na mulher com hipertensão há 2,2 vezes mais chances de ocorrência de PTBPN do que em um indivíduo que não apresenta essa condição clínica $(\mathrm{OR}=2,2,95 \% \mathrm{IC}, 1.11$ a 4.30$)$. A chance de uma mulher usuária de droga ilícita ter um PTBPN é 7,2 vezes maior do que de uma mulher que não-usuária. Na avaliação do fator diabetes, o teste Exato de Fischer não demonstrou diferença significativa (TAB. 1).

Em relação à influência da idade da parturiente na ocorrência do PTBPN pode-se demonstrar, através da aplicação do teste $\mathrm{t}$ de Student, uma diferença estatisticamente significativa $(p=0,010)$ entre os grupos com ocorrência de PTBPN e os grupos de parto a termo. $O$ grupo que teve PTBPN é significativamente mais jovem.

\section{Análise de regressãa logística}

Após a análise dos fatores de risco estudados, apenas o consumo de drogas ilícitas permaneceu significativo para a ocorrência de PTBPN. Com base no modelo de regressão logística, observa-se que uma mulher usuária de drogas ilícitas durante a gestação tem $66,7 \%$ de chances para PTBPN, enquanto uma não-usuária tem $25 \%$.

\section{DISCUSSÃO}

Vários estudos têm apresentado os prováveis mecanismos biológicos que reforçam a possibilidade de a doença periodontal representar um fator de risco para a ocorrência de PTBPN. Gibbs et al. (1992) correlacionam as infecções sub- clínicas com a ocorrência de parto prétermo. Os mecanismos propostos analisam no âmbito bioquímico o papel das prostaglandinas e dos produtos bacterianos e os relacionam de maneira positiva com os eventos etiopatogênicos encontrados na doença periodontal e no parto pré-termo.

Offenbacher et al. (1996) consideraram a presença de infecção periodontal em mulheres grávidas um fator de risco até então desconhecido para a ocorrência de PTBPN. Na mesma linha, Offenbacher et al. (1998) demonstraram o aumento nos níveis de PGE2 e IL-lb no fluido gengival de mulheres que apresentaram PTBPN em comparação com as mulheres de período gestacional normal. Hasegawa et al. (2003) demonstraram que as mulheres com ameaça de parto pré-termo apresentaram pior condição periodontal e níveis de IL-8 e IL-1ß séricos elevados em comparação com aquelas com parto a termo.

Offenbacher et al. (1998) e MitchellLewis et al. (2001) fizeram análises microbiológicas e identificaram maior ocorrência de periodontopatógenos na placa subgengival das mulheres que tiveram parto pré-termo do que na placa subgengival das mães que deram à luz bebês em parto a termo e com peso normal. Entretanto, Noack et al. (2005) e Jarjoura et al. (2005) não observaram diferença significativa no aspecto microbiológico entre os grupos com e sem prematuridade.

Estudos clínicos epidemiológicos têm demonstrado uma relação significativa entre a ocorrência de PTBPN e o aumento da gravidade da doença periodontal (DASANAYAKE, 1998; DAVENPORT et al., 1998; JEFFCOAT et al., 2001; LOPEZ et al., 2002a; OFFENBACHER et al., 1998; RADNAI et al., 2004; JARJOURA et al., 2005; KHADER e TA' ANI, 2005).

Por um lado, a prevalência da PTBPN encontrada no presente estudo pode ser considerada elevada $(26,7 \%)$ em comparação com os valores mencionados

\begin{tabular}{llll}
\multicolumn{5}{c}{ TABELA 1 } \\
\multicolumn{4}{c}{ Fatores de risco clássicos para a ocorrência de PTBPN } \\
\hline Fatores de risco & PTBPN & Parto a termo & Valor de $\boldsymbol{p}$ \\
\hline Hábito de fumar & $28,6 \%$ & $26,1 \%$ & $p=0,720$ \\
Consumo de bebida alcoólica & $31 \%$ & $25,8 \%$ & $p=0,554$ \\
História pregressa de PTBPN & $22,7 \%$ & $27,3 \%$ & $p=0,538$ \\
Infecção geniturinária & $30,8 \%$ & $23,9 \%$ & $p=0,241$ \\
Diabetes & $21,4 \%$ & $26,9 \%$ & $p=0,765$ \\
Hipertensão & $39,6 \%$ & $22,9 \%$ & $p=0,016$ \\
Uso de droga ilícita & $71,4 \%$ & $25,8 \%$ & $p=0,017$ \\
\hline
\end{tabular}


por Willians et al. (2000) 11\%; MitchellLewis et al. (2001) 16,5\%; López et al. (2002a) 4,7\%. Por outro lado, está de acordo com a variação na prevalência de 10 a $40 \%$ citada por Correa (1983) e explicada por razões raciais, condições socioeconômicas, região geográfica e carência de atendimento pré-natal. Cabe ressaltar que esta investigação foi desenvolvida em uma maternidade considerada como unidade de referência regional para gravidez de alto risco, justificando a ocorrência elevada de PTBPN.

Através da análise univariada e do modelo de regressão logística multivariada, este estudo avaliou a associação não só da condição periodontal mas também de outros fatores de risco considerados "clássicos" para a ocorrência de PTBPN. Os resultados demonstraram que na análise univariada os fatores consumo de drogas ilícitas, hipertensão e idade apresentaram significância estatística, e somente a variável consumo de drogas ilícitas permaneceu com significância estatística para a ocorrência de PTBPN, após a aplicação do modelo de regressão logística $(p=0,0419)$. O percentual de chances para a ocorrência de PTBPN foi de 66,7\% nas usuárias de drogas ilícitas durante a gestação e $25 \%$ nas não-usuárias.

No presente estudo analisou-se a condição periodontal materna em relação à gengivite e à periodontite. Pôde-se demonstrar que, mesmo quando as gestantes foram estratificadas de acordo com a gravidade, a doença periodontal não apresentou uma relação estatisticamente significativa com a ocorrência de PTPBN. Esses dados confirmam os resultados dos estudos de Mitchell-Lewis et al. (2001), Davenport et al. (2002), Moore et al. (2005) e Noack et al. (2005).

A prevalência de periodontite encontrada neste estudo $(36,1 \%)$ é semelhante à reportada por outras investigações, a saber: Davenport et al. (2002) 44\%; López et al. (2002a) 36,4\%; Jarjoura et al. (2005) $30,1 \%$ - embora tenham sido utilizados critérios diferentes para a definição de caso doença periodontal.

Na literatura são conflitantes as inferências sobre a associação da infecção periodontal com a ocorrência de PTBPN, por isso devem ser analisadas com cautela. A variabilidade dos indivíduos constituintes da amostra nos estudos e a utilização de diversos critérios para a definição de um caso doença periodontal podem não apenas representar fatores dificultadores para comparação dos resultados mas também levar a interpretações equivocadas. Assim, outros estudos com critérios semelhantes são necessários para aprofundar o conhecimento da associação entre a infecção periodontal e a ocorrência PTBPN.

\section{CONCLUSÃO}

Através da metodologia utilizada neste estudo, pôde-se concluir que, na amostra avaliada, a condição clínica periodontal materna não apresentou uma associação estatisticamente significativa com a ocorrência de PTBPN.

\section{ABSTRACT}

The purpose of this study was to assess the association of the maternal periodontal condition upon occurrence of preterm birth and low birth weight (PTB/ LBW). Participated in this study, 236 women at an average of 25 years of age, with similar socio-economic conditions, who had given birth in public health institutions in Belo Horizonte, MG, Brazil. Maternal, obstetric and pediatric data were collected from medical records. During post-partum hospitalization, the periodontal examination was performed on full-mouth at six sites per tooth and included the clinical parameters of probing depth (PD), clinical attachment level (CAL), and bleeding on probing (BOP). For the purpose of analysis, the presence of one site per tooth with PD and CAL e" $4 \mathrm{~mm}$ indicated a case of periodontal disease. There were no significant differences regarding analyses of variables: race, level of education, previous pre-term, diabetes, genitourinary infection, smoking habits, alcohol consumption, and periodontal disease. The chi-square test revealed a statistical significance for risk factors: hypertension $(p=0.016)$ and consumption of illicit drugs $(p=0.017)$. The Student t-test showed a statistical significance in age $(p=0.010)$. In the multivariate logistic regression analysis, only the consumption of illicit drugs was significant, increasing the occurrence of PTB/ LBW from $25 \%$ to $66.7 \%$. These results demonstrate that the maternal periodontal disease presented no statistically significant association with the occurrence of PTB/LBW ( $p=0.364)$.

\section{KEY WORDS}

Periodontal diseases, adverse effects. Preterm birth. Infant low birth weight. Pregnancy, risk factors.

\section{REFERENCIAS}

AMERICAN ACADEMY OF PERIODONTOLOGY. Parameters of Care. J. Periodontol., Chicago, v. 71, no.5, p.853-858, Suppl. May 2000.
COLLINS, J. G. et al. Effects of a Porphyromonas gingivalis Infection on Inflammatory Mediator Response and Pregnancy Outcome in the $\mathrm{Ha}-$ mster. Infect. Immun., Washington, v.62, no.10, p.4356-4361, Oct. 1994.

COLLINS, J. G. et al. Experimental Periodontitis Retards Hamster Fetal Growth. J. Dent. Res., Alexandria, v.74, Spec. Issue, p.158, 1995. Abs \# 1171.

CORREA, M. D. Noções Práticas de Obstetrícia. Belo Horizonte: Cultura Médica, 1983. Cap. 8, Parto Prematuro, p. 67-74.

DASANAYAKE, A. P. et al. The Association between Porphyromonas gingivalis; Specific Maternal Serum IgG and Low Birth Weight. J. Periodontol., Chicago, v.72, no.11, p.1491-1497, Nov. 2001.

DASANAYAKE, A. P. Poor Periodontal Health of the Pregnant Woman as a Risk Factor for Low Birth Weight. Ann. Periodontol., Chicago, v.3, no.1, p.206-212, 1998.

DAVENPORT, E. S. et al. The East London Study of Maternal Chronic Periodontal Disease and Preterm Low Birth weight Infants: Study Design and Prevalence Data. Ann. Periodontol., Chicago, v.3, no.1, p.213221, July 1998.

DAVENPORT, E. S. et al. Maternal Periodontal Disease and Preterm Low Birthweight: Case Control Study. J. Dent. Res., Alexandria, v.81, no.5, p.313-318, May 2002.

GIBBS, R. S. et al. A Review of Premature Birth and Subclinical Infection. Am. J. Obstet. Gynecol., New York, v.166, no.5, p.1515-1528, May 1992.

HASEGAWA, K. et al. Associations between Systemic Status, Periodontal Status, Serum Cytokine Levels, and Delivery Outcomes in Pregnant Women with a Diagnosis of Threatened Premature Labor. J. Periodontol., Chicago, v.74, no.12, p.17641770, Dec. 2003.

HILL, G. B. Preterm Birth: Associations with Genital and Possibly Oral Microflora. Ann. Periodontol., Chicago, v.3, no.1, p.222-232, July 1998. 
JARJOURA, K. et al. Markers of Periodontal Infection and Preterm Birth. Am. J. Obstet. Gynecol., New York, v.192, no.2, p.513-519, Feb. 2005.

JEFFCOAT, M. K. et al. Periodontal Infection and Preterm Birth. Results of a Prospective Study. J. Am. Dent. Assoc., Chicago, v.132, no.7, p.875880, July 2001 .

JEFFCOAT, M. K. et al. Periodontal Disease and Preterm Birth: Results of Pilot Intervention. J. Periodontol., Chicago, v.74, no.8, p.12141218, Aug. 2003.

KHADER, Y.S; TA'ANI, Q. Periodontal Diseases and Risk of Preterm Birth and Low Birth Weigth: A MetaAnalysis. J. Periodontol., Chicago, v.76, no.2, p.161-165, Feb. 2005.

LOPEZ, N. J.; SMITH, P. C.; GUTIERREZ, J. Higher Risk of Preterm Birth and Low Birth Weight in Women with Periodontal Disease. J. Dent. Res., Alexandria, v. 81, no.1, p.58-63, Jan. 2002a.

LOPEZ, N. J.; SMITH, P.C.; GUTIERREZ, J. Periodontal Therapy May Reduce the Risk of Preterm Low Birth Weight in Women with Peridontal Disease: A Randomized Controlled Trial. J. Periodontol., Chicago, v.73, no.8, p.911-924, Aug. 2002b.

LOPEZ, N. J. et al. Periodontal Therapy Reduces the Rate of Preterm Low Birth weight in Women with Pregnancy-Associated Gingivitis. J. Periodontol., Chicago, v.76, no.11, p.2144-2153, Suppl. Nov. 2005.

McCORMICK, M. C. The Contribution of Low Birth Weight to Infant Mortality and Childhood Morbidity. N. Engl. J. Med., Royston, v.312, no.2, p.82-90, Jan. 1985.
MITCHELL-LEWIS, D. et al. Periodontal Infections and Pre-Term Birth: Early Findings from a Cohort of Young Minority Women in New York. Eur. J. Oral Sci., Copenhagen, v.109, p.34-39, 2001.

MOORE, S.; RANDHAWA, M.; IDE, M. A Case-Control to Investigate an Association between Adverse Pregnancy Outcome and Periodontal Disease. J. Clin. Periodontol., Copenhagen, v.32, no.1, p.1-5, Jan. 2005.

NOACK, B. et al. Periodontal Status and Preterm Low Birth Weight: A Case Control Study. J. Periodontal Res., Copenhagen, v.40, no.4, p.339345, Aug. 2005.

OFFENBACHER, S. et al. Periodontal Infection as Possible Risk Factor for Preterm Low Birth Weight. J. Periodontol., Chicago, v.67, no.10, p.1103-1113, Suppl., Oct. 1996.

OFFENBACHER, S. et al. Potential Pathogenic Mechanisms of Periodontitis-Associated Pregnancy Complications. Ann. Periodontol., Chicago, v.3, no.1, p.233-250, July 1998.

RADNAI, M. et al. Possible Association between Preterm Birth and Early Periodontitis: Pilot Study. J. Clin. Periodontol, Copenhagen, v.31, no.9, p.736 -741, Sept. 2004.

SCANNAPIECO, F. A.; BUSH, R. B.; PAJU, S. Periodontal Disease as a Risk Factor for Adverse Pregnancy Outcomes. A Systematic Review. Ann. Periodontol., Chicago, v.8, no.1, p.70-78, Dec. 2003.

WILLIANS, C. et al. Mechanisms of Risk in Preterm Low-Birthweight in Infants. Periodontology 2000, Munksgaard, v.23, no. 7, p.142-150, June 2000.
WORLD HEALTH ORGANIZATION. Consultation on Definitions and Standards Related to Maternal and Child Health and the Perinatal Period. Washington, p.2, 1987.

\section{AGRADECIMENTOS}

Ao Corpo Clínico da Maternidade Escola Hilda Brandão da Santa Casa de Belo Horizonte. Ao Fundo de Incentivo à Pesquisa da Pontifícia Universidade Católica de Minas Gerais (Projeto FIP 2001/04-TLE).

Endereço dos autores

Alcione Maria Soares Dutra de Oliveira

Rua Maranhão, 938 / 1001

30150-3330 - Belo Horizonte - Minas Gerais

Tel: (31) 3225.4378 / Fax: (31) 3241.4083

E-mail: petdutra@terra.com.br

Peterson Antônio Dutra de Oliveira Rua Maranhão, 938 / 1001

30150-3330 - Belo Horizonte - Minas Gerais

Tel: (31) 3225.4378 / Fax: (31)

3241.4083

E-mail: petdutra@terra.com.br

Fernando de Oliveira Costa

Rua Trieste, 323

31340-430 - Belo Horizonte - Minas

Gerais

Tel: (31) 3491.3000 / (31) 3284.2466

E-mail: focperio@uol.com.br

Flávio Ricardo Manzi

Rua Napoleão Laureano, 161 - Vila Independência

13418-060 - Piracicaba - São Paulo

E-mail: manzi@pucminas.br

Maurício Greco Cosso

Rua Progresso, 290 - Padre Eustáquio 30720-320 - Belo Horizonte - Minas Gerais

E-mail: mauriciocosso@uaigiga.com.br 